Provided by the author(s) and University of Galway in accordance with publisher policies. Please cite the published version when available.

\begin{tabular}{|c|c|}
\hline Title & $\begin{array}{l}\text { In-game motion dynamics provide a means of exploring the } \\
\text { cognitive dynamics of deception }\end{array}$ \\
\hline Author(s) & $\begin{array}{l}\text { O’Hora, Denis; Redfern, Sam; Duran, Nicholas; Zgonnikov, } \\
\text { Arkady; Sweeney, Daragh }\end{array}$ \\
\hline $\begin{array}{c}\text { Publication } \\
\text { Date }\end{array}$ & 2018-07-15 \\
\hline $\begin{array}{l}\text { Publication } \\
\text { Information }\end{array}$ & $\begin{array}{l}\text { O'Hora, Denis , Redfern, Sam, Duran, Nicholas, Zgonnikov, } \\
\text { Arkady, \& Sweeney, Daragh. (2018). In-game motion } \\
\text { dynamics provide a means of exploring the cognitive dynamics } \\
\text { of deception. Paper presented at the IEEE Games, } \\
\text { Entertainment and Media Conference (GEM) 2018, Galway, } \\
\text { Ireland, 15-17 August. }\end{array}$ \\
\hline Publisher & IEEE \\
\hline $\begin{array}{l}\text { Link to } \\
\text { publisher's } \\
\text { version }\end{array}$ & http://sites.ieee.org/ieeegem/ \\
\hline Item record & http://hdl.handle.net/10379/14634 \\
\hline
\end{tabular}

Downloaded 2023-04-26T12:53:05Z

Some rights reserved. For more information, please see the item record link above.

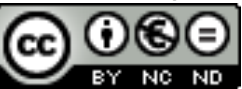




\title{
In-game motion dynamics provide a means of exploring the cognitive dynamics of deception
}

\author{
Denis O’Hora*, Sam Redfern ${ }^{\dagger}$, Nicholas Duran ${ }^{\ddagger}$, Arkady Zgonnikov ${ }^{\S}$, and Daragh Sweeney ${ }^{\dagger}$ \\ ${ }^{*}$ School of Psychology, National University of Ireland Galway \\ University Road, Galway, Ireland \\ Email: denis.ohora@nuigalway.ie \\ ${ }^{\dagger}$ School of Engineering and Informatics, National University of Ireland Galway \\ University Road, Galway, Ireland \\ Email: sam.redfern@nuigalway.ie, D.SWEENEY7@nuigalway.ie \\ ${ }^{\ddagger}$ School of Social and Behavioral Sciences, Arizona State University, Glendale, Arizona, USA \\ Email: nicholas.duran@asu.edu

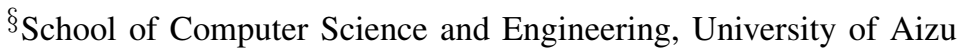 \\ Aizuwakamatsu, Fukushima, Japan \\ Email: arkady@u-aizu.ac.jp
}

\begin{abstract}
User interfaces that produce an immersive and intuitive in-game experience depend on a strong coupling between user input and the motion of game objects. Such user interfaces require a high sensitivity to user movement that has the potential to reveal characteristics of user cognitive processes that occur during gameplay. The current project investigates whether cognitive processing during deception affects in-game motion. We present here two paradigms that allow deception to be expressed over repeated trials and in a naturalistic setting. The first, an interactive exhibit at Science Gallery Dublin ${ }^{\mathrm{TM}}$, tracked motion while users deceptively responded to autobiographical statements. The second, a two-player bluffing game, tracked motion during unsanctioned, motivated deception.

Our findings indicate that in-game motion is influenced by the cognitive processes underlying deception. In-game motion provides an important source of data on human psychological processes that can stimulate theoretical progress within psychology and contribute to the development of more credible artificial agents.
\end{abstract}

\section{INTRODUCTION}

\section{A. Movement in Computer Games}

A user interface that 'just works' is a prerequisite for an enjoyable game. When user inputs guide the motion of an ingame object, such as a sprite, then that object should move as we expect to. More technically, for in-game motion to feel intuitive, a strong coupling between a user's motor output and in-game motion must be established and maintained. Indeed, in Calleja's [1] model of digital game involvement, accommodating these expectations is a central feature: "Planned motion is made manifest by the controlled agent(s) creating a potential for action defined by the movement affordances designed into the virtual environment or world" (p. 85). When the relationship between user movement and in-game motion is undermined or uncertain (e.g., through laggy connections), it can generate strong negative reactions from the user. With this in mind, crowdsourced player telemetry (the gathering of in-game motion and other behavioral data from thousands of users as they play the games) is now routinely used for marketing and pre/post-release product improvement [2].

Coupling between user input and the motion of game objects depends on interfaces that are highly sensitive to motor input. Psychological research has highlighted that cognitive processing influences motor output in a relatively continuous fashion. Consequently, in-game motion may provide a sensitive index of a player's unfolding cognitive processes. In this paper, we introduce two novel experimental paradigms that take advantage of in-game motion for capturing and evaluating sanctioned and unsanctioned deception. We begin by contextualizing this work in the latest psychological research on the intricate relationship between cognition and action and current psychological understandings of deception. We then report analysis of the data collected at scale from participants "in the wild," and end with a discussion on how these simple experimental paradigms can be used to make further in-roads in understanding the psychological aspects of deception and game design in general.

\section{B. Laboratory Analyses of Movement during Cognition}

Based on insights from dynamical systems and motor control theorists, cognitive processing is taken to be inextricably intertwined with the continuous movements of the body [3], [4]. Even in simple laboratory tasks, periods of instability and flow in processing can be mapped onto unique signatures of movement. Moreover, response behaviors are also expressions of emergent order that are constrained and guided by task conditions that continuously evolve over varied time scales [5], [6]. Given this relationship between mind, body, and environment, one way to exploit the inherent co-variation is with the use of game-like, motion-rich cognitive tasks. For example, in the "visual world paradigm," the motion of the hand is tracked during the selection of visually presented "targets" amidst visually co-present "distractors" on a computer screen. By surreptitiously recording the position of the hand during 
the movement (via the position of a cursor on the computer screen), the researchers gain access to the hand trajectories, which can reveal how information is being managed in realtime.

To provide a tangible example, McKinstry et al. [7] had participants evaluate the truth of simple propositions by navigating a computer mouse to true or false response options in the uppermost corners of a screen. Propositions that had a high level of uncertainty of being true, such as "Is murder sometimes justifiable?", were answered with slower hand movements and with greater moment-to-moment fluctuations than propositions with a high level of certainty (e.g., "Should you brush your teeth everyday?"). These unstable hand movements suggest that greater cognitive effort is involved in evaluating ambiguously true information. Moreover, the continuous movements of the hand reveal an immediate and persistent influence of a "positive confirmation" bias throughout the response (see also [8]). This confirmation bias was most salient while answering "no" to propositions that were clearly false, such as "Is the mother younger than the daughter?". For these propositions, there was a tendency for the hand to gravitate relatively slowly toward a "yes" response (positive confirmation) during a "no" response movement. This pattern of movement suggests that both target false response and a competitor confirmation bias were simultaneously activated, with the activation from the target response eventually prevailing.

\section{Deception: import and cognitive basis}

From "white lies" to more nefarious intent, lying occurs at a surprisingly high rate in everyday interactions [9], [10]. In most cases, deception is minimally consequential, with the most severe risk being a loss of social standing. Nevertheless, in other cases, as with insider threats in corporate settings, or malingering in health evaluations, the consequences can be dire. It is no surprise then that a great deal of effort has gone into developing deception detection techniques that are accurate, robust, and easily deployable. One major direction in this research has been to take advantage of the assumed increase in arousal and stress associated with deception, as evidenced by the polygraph, to more modern attempts at voice analysis [11], [12]. However, arousal and stress can easily occur when individuals are telling the truth; moreover, highly motivated deceivers might be particularly skilled at masking deceptive responses [13]. For this reason, there has been a recent turn towards underlying mechanisms of deception that might prove more difficult to overcome, specifically, the increased cognitive demands imposed by deception that are not present with the truth [14], [15].

One reason deception is held to be more challenging is due to the so-called cognitive executive function processes that are generally implicated in the control and coordination of mental activity. These processes include skills like ignoring distractor stimuli in the environment while attending to a primary goal [16], [17], and the efficient allocation of attentional resources that permit rapid transitions between tasks [18], [19]. Indeed, deception requires the ability to ignore a distractor — the truth - from being expressed, while simultaneously consulting the truth to select and deliver a convincing lie [20], [21]. Many researchers interested in building deception detection tools have taken note that one of the consequences of these increased cognitive demands is that it results in increased time to make a response [22], [23]. Based on a recent meta-analysis of 114 studies that examined the time taken to lie in controlled computerized tasks, the relationship appears to be broadly consistent [24]. Nevertheless, for many researchers outside the arena of deception, response time is considered a coarse and indirect index of underlying cognitive processes. This view is uniquely addressed within a research domain known as "action dynamics".

\section{Deception in movement}

Most cognitive theories of deception assume the underlying processes occur as a series of discrete and minimally interactive ordered components, in which one component gives way to the next in a single chain of processing [25]. An alternative to this view, supported by the action dynamics perspective, is that the underlying activations of true and false information occur as partially overlapping and distributed mental states that compete with each other over time. This notion of "over time" is critical as it is these moments of processing that are often obscured by the ballistic movements used to capture response times (e.g., the time taken to make a key press), which effectively collapses intermediary cognitive processes to a single discrete behavior [26], [27]. In contrast, the promise of the action dynamics approach is that the competition between false responding and the truth will be revealed in the unfolding "micro-behaviors" within response execution, and importantly, this new information will improve our abilities to detect deception.

Early support for a dynamical perspective has been shown in the work of Duran et al. [28] who used a version of the aforementioned visual world paradigm. In their study, participants had to choose between between "yes" and "no" options on a screen in response to statements about experiences they may or may not have had. These statements varied in terms of their likelihood of being common experiences for most people. Critically, participants were randomly prompted to tell the truth on half the statements and to provide false answers on the other half. Based on analysis of the response movements, this study has found clear signatures for graded activation biased toward true and false responses depending on the intended answer. When providing false answers, there was an early and persistent activation elicited by the truth that was particularly pronounced when the target answer was "yes." Similar findings have also been demonstrated in similar motion capture setups, such as those examining how people falsely reject having knowledge about critical aspects of a scenario that would expose them as having had cheated [29] or possessing a false identity [30]. 


\section{E. Interactive games as means to investigate deception}

To date, analyses of movement during deception has occurred in tightly controlled but unrealistic laboratory settings or in real-world settings that require time-consuming and subjective behavioral ratings. Both laboratory and field studies also tend to rely on overly discrete behaviors that may not be the strongest predictors of deception, although the action dynamics approach in laboratory studies does allow the measurement of relatively continuous motion that might better map onto the cognitive complexities of lying. To find a compromise between laboratory and field studies, interactive games might be ideal as they can gather continuous movement data in a naturalistic setting. In addition, when one considers the amount of time that users play, e.g., online poker, deception games that are engaging have the potential to collect vast amounts of player movement telemetry.

In what follows, we describe two experimental paradigms designed to exploit player movement telemetry to investigate deception. The first paradigm, Lie2Me, was an interactive touchscreen exhibit at Science Gallery Dublin ${ }^{\mathrm{TM}}$. Patrons at the gallery were presented with autobiographical questions and were instructed to lie when answering half the questions. The second paradigm, Fool Me Once!, was an online two-player bluffing card game, in which players won points by deceiving their opponent or spotting their opponent's deception.

\section{Paradigm 1 - Lie2Me}

\section{A. Method-Lie2Me}

1) Setting and Recruitment: Participants took part in the Lie2Me exhibit at the Science Gallery Dublin ${ }^{\mathrm{TM}}$, Trinity College Dublin, in the first month of the FAKE exhibition (08/03/2018 to 08/04/2018). All data were collected in accordance with procedures approved by the Research Ethics Committee at Science Gallery, and informed consent was obtained from all participants. The FAKE exhibition (https://dublin.sciencegallery.com/fake/) addressed issues of fakery and deception across a variety of interactive educational exhibits. Interested patrons were provided information on all the experiments and were asked to sign a consent form by a member of Science Gallery staff prior to participation. Patrons who registered were allocated a participation identification number (PID) and asked to provide limited demographic information. Patrons were continuously monitored by Science Gallery staff in case the behavioral experiments caused any offense or discomfort. No adverse reactions to Lie2Me were reported.

During the first month of the exhibit, 324 patrons played Lie2Me providing their PIDs. Demographic data have not yet been released for those who participated in $\mathrm{Lie} 2 \mathrm{Me}$, but patrons of the Science Gallery typically range in age from 12 to 65 , with the greatest concentration between 18 and 30; the gender distribution of visitors is in line with Irish society at large (see [31] for representative demographics of Science Gallery attendees).

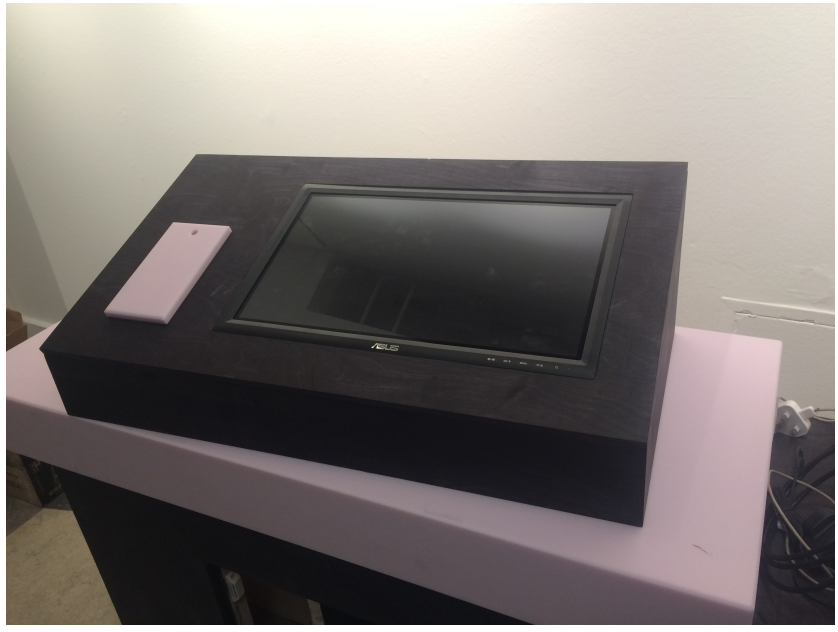

Fig. 1. Lie2Me exhibit at Science Gallery Dublin ${ }^{\mathrm{TM}}$

2) Hardware: Lie2Me was set up as a touchscreen-only exhibit (see Figure 1). The touchscreen was set in a tabletop structure such that the touchscreen seemed part of the top of the structure. A pink panel was presented to the left of the touchscreen to house sensors for measurement of galvanic skin response (GSR), but this feature was not implemented during the first month of the exhibition. The touchscreen was ASUS VT207N 19.5 inch monitor, with a resolution of $1600 \times 900$ pixels and refresh rate of $60 \mathrm{~Hz}$. Participants responded while sitting at a stool next to the exhibit.

3) Software: Lie2Me was written using HTML5 and Javascript for the front-end, with data submitted via Ajax to a remote MySQL database for secure storage and analysis. Finger location during responses to trials was recorded every time the $\mathrm{x}, \mathrm{y}$ position of the cursor changed. Recording intervals indicated a cursor sampling rate of approximately $60 \mathrm{~Hz}$. Although Lie2Me is a one-player game without any game design reasons for network connectivity, one of our considerations was that it would be deployed as a public installation in the Science Gallery in Dublin while the technical research team are in Galway. Our technology choices meant that remote updates of the system was very straightforward and did not require intervention from Science Gallery staff.

4) Procedure: To begin Lie2Me, participants approached the exhibit and entered their PID using a touchscreen keypad. Interaction with the game was solely via touchscreen. Following a short instructional video, participants completed 32 trials in which they were asked to tell the truth or lie when asked a biographical question (see Figure 2), by dragging and dropping a small red ball on the screen to either the word "Yes" or "No" at the top of the screen. Finally, the participants completed a questionnaire containing the same 32 questions to identify any failures to lie or tell the truth during the game.

In each trial, the word "Truth" in blue or "Lie" in red appeared first at the top of the screen. Next, the words of a statement appeared at a rate of one word every $500 \mathrm{~ms}$ (e.g., "Have $(500 \mathrm{~ms})$ you $(500 \mathrm{~ms})$ met..."). The final word did not 
appear until the participant moved the response ball out of the starting area at the bottom of the screen. The statements were generated based on the questionnaire previously used in similar studies [28]. The statements varied in the probability of responding "yes" truthfully from low (e.g., "Have you ever met Messi?") to high (e.g., "Have you ever eaten pizza?" ). Half of the statements referred to observations (e.g., "Have you seen a movie?") and half referred to actions (e.g., "Have you built a robot?'”). The instruction to tell the truth or lie was randomly generated with the constraint that each participant was prompted to lie and tell the truth exactly half the time. The locations of "Yes" and "No" at the top of the screen were randomly assigned for each participant. At the end of the game, participants were shown their response trajectories for truths and lies so that they could see how deception is expressed in their behavior. Minimal debriefing was provided afterward on screen. Further debriefing materials were made available via email to participants.

5) Exclusion Criteria: Prior to analysis, we applied a number of exclusion criteria designed to exclude participants who did not pay due attention to the experimental task. From a total of 324 participants, trials that satisfied the following criteria were excluded: trials with a reaction time greater than $5 \mathrm{~s}$, trials with pauses longer than $1.5 \mathrm{~s}$ and trials with fewer than 30 sampled coordinates. Participants with fewer than 24 trials remaining after these exclusions were removed altogether. Overall, 257 participants were retained with at least 24 trials each.

\section{B. Results - Lie2Me}

1) Trajectory Measures: Visual inspection of the response trajectories revealed that lies tend to be more deflected than truths (Figure 3 ). In order to statistically analyze the effects of lying and truth-telling on trajectories, we extracted three wellestablished measures (Figure 4) from the response trajectories using the $\mathrm{R}$ package mousetrap [32]. Response time was the time elapsed from the point that the participant moved the response ball out of the starting area ("exit point") until entering a response area (either "Yes" or "No"). Deflection towards the unchosen option was measured using maximum absolute deviation (MAD), the greatest perpendicular distance from a straight line connecting the exit point to the final point in the trajectory. Vacillation was measured using $x$-flips. Xflips refer to changes in the horizontal direction of a trajectory (e.g., from moving left to moving right). A flip threshold of 2 pixels was employed to exclude irrelevant jitter in the trajectory.

In line with the previous studies [28], when participants responded truthfully, they responded faster, with less deflection and vacillation than when they lied (Figure 5). However, this effect was strongly moderated by the answers they were giving. When participants responded "Yes" to a statement, it was very easy to distinguish truths from lies. True responses to "Yes" induced the fastest, most direct and least complex trajectories. In contrast, false responses to "Yes" were the
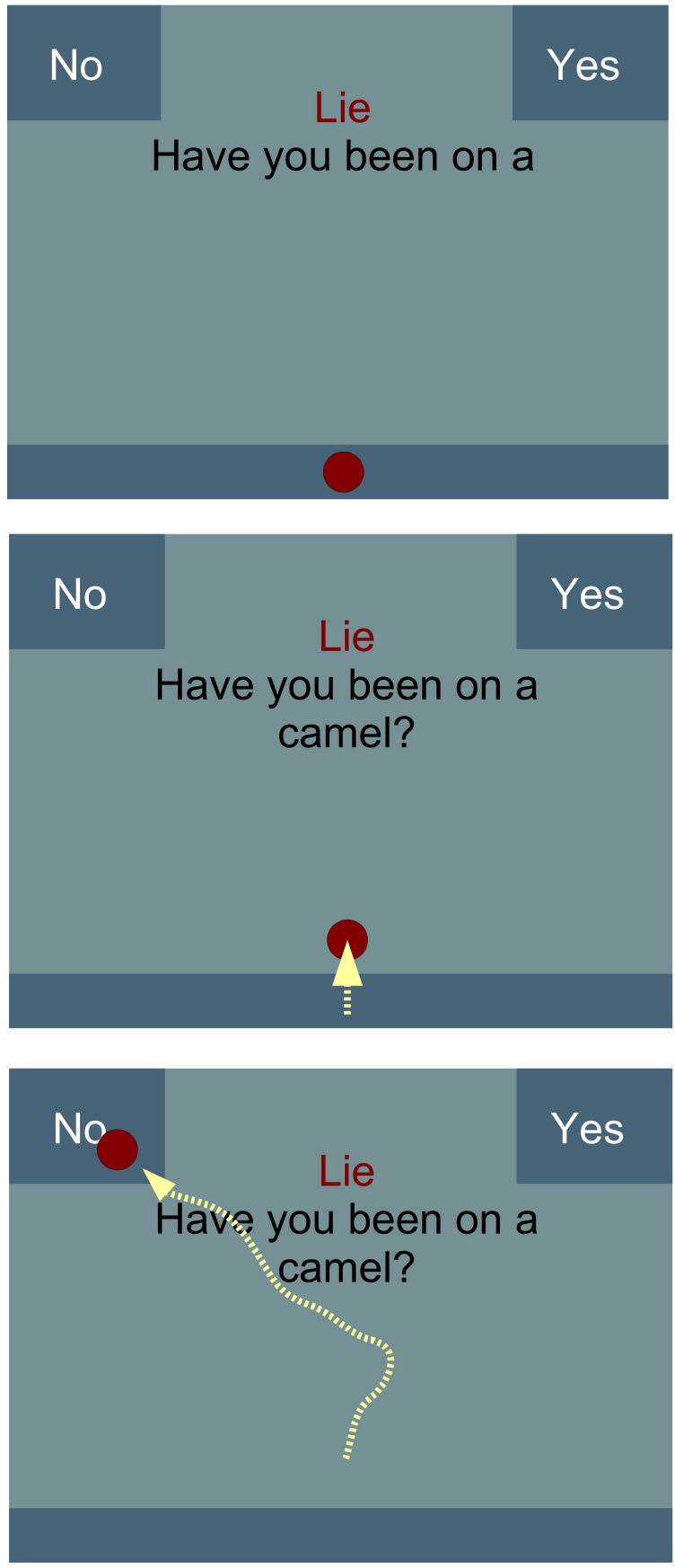

Fig. 2. Lie2Me Procedure

slowest, least direct and more complex responses. For "No" responses, lies and truths looked similar.

2) Average Trajectories: To provide a visualization of the how participants moved during deception, we generated average trajectories. To do this, the $\mathrm{x}$ - and $\mathrm{y}$-components of each trajectory were interpolated into 101 time steps (see [34], [32] for details). Next, the trajectories of "Yes" decisions reaching to the left-hand response area and "No" decisions to the righthand response area were reflected across the vertical axis at the origin. As a result, all "Yes" trajectories ended in the top 


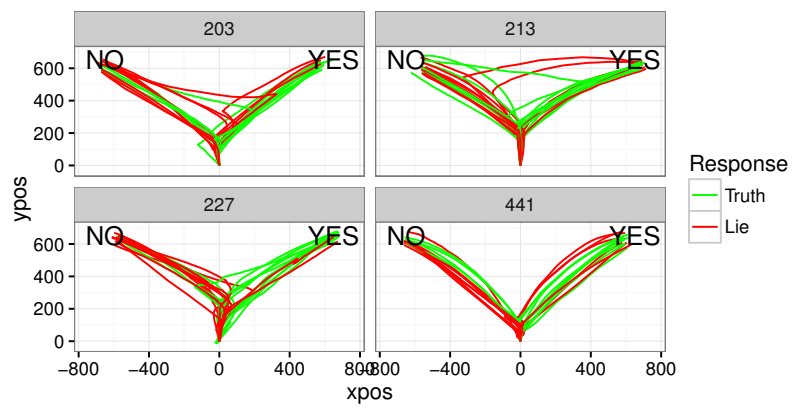

Fig. 3. Examples of Lie2Me trajectories from four randomly chosen participants

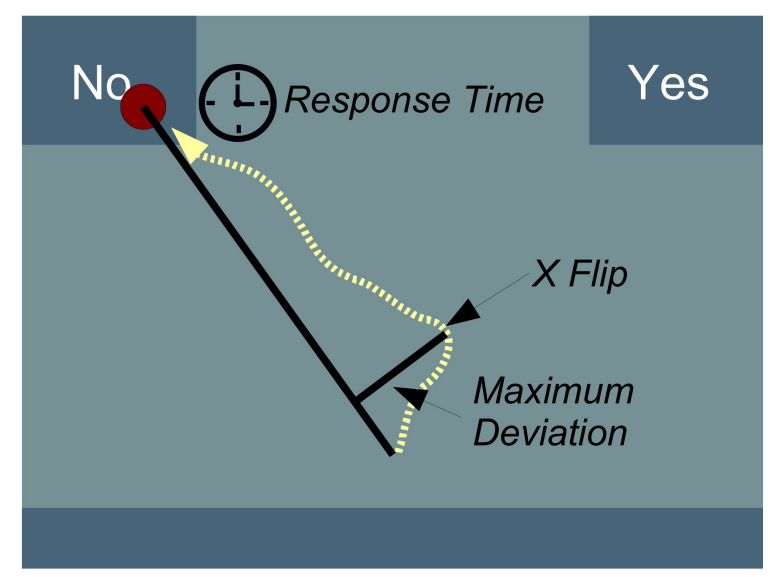

Fig. 4. Lie2Me trajectory measures

right-hand corner while all "No" responses ended in the top left-hand corner.

The average trajectories reinforce the findings obtained from the trajectory measures (Figure 6). Deceptive "Yes" responses are easily distinguishable from true "Yes" responses, but deceptive and true "No" responses look similar.

\section{Paradigm 2 - Fool Me Once!}

Lie2Me was quite similar to a laboratory psychology paradigm. A strength of this approach is that the data can be easily treated using typical psychological techniques. A weakness of the approach is that the ecological validity of the paradigm as an assessment of real-life deception remains quite low. When individuals lie, they typically choose to do so themselves, they are not instructed to lie as they were in Lie2Me. The second paradigm we outline, Fool Me Once!, is much closer to a real-life situation in that it took the form of a simple online player-versus-player (PvP) card game. Bluffing is an integral part of $\mathrm{PvP}$ games in general and arises naturally. In Fool Me Once!, we made bluffing a core mechanic of the game and tracked in-game movement during bluffing to establish whether there were typical movement features observed.
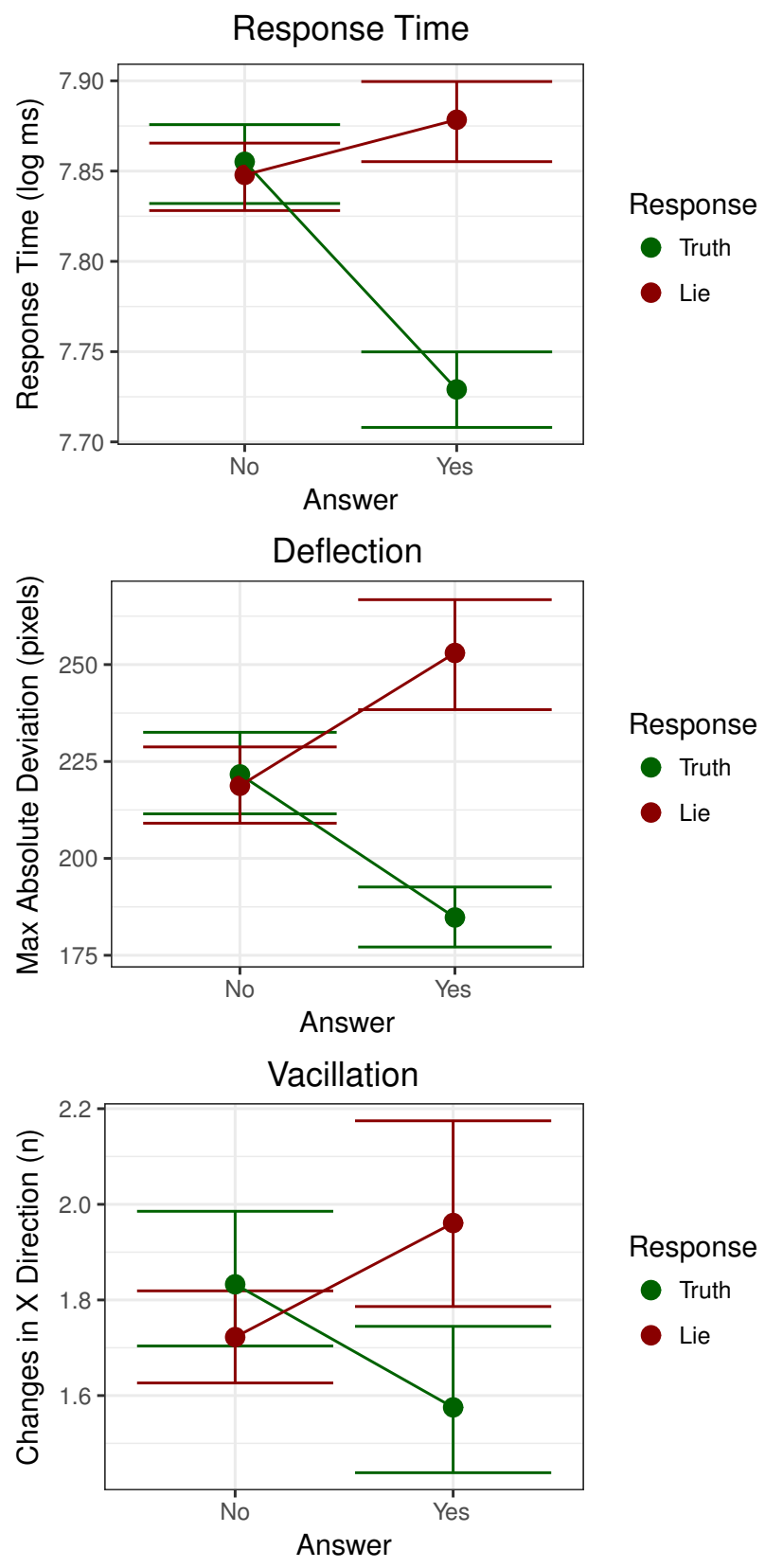

Fig. 5. Effects of Deception and Answer on Lie2Me Trajectory Measures. Plots developed using ggplot2 [33]

\section{A. Method - Fool Me Once!}

1) Setting: Our intention is to eventually deploy Fool Me Once! online to be played wherever users happen to be. For the initial testing stages, we recruited two sets of pilot participants to play the game in a computer lab to extract preliminary data.

2) Game Design: Fool Me Once! is a stripped-down bluffing card game based on the "Higher or Lower" or "Play Your Cards Right" game. In the original "Higher or Lower" game, players bet on whether the next card to be revealed will be higher or lower than a known card. In Fool Me Once!, two players play against one another, taking alternate turns as 


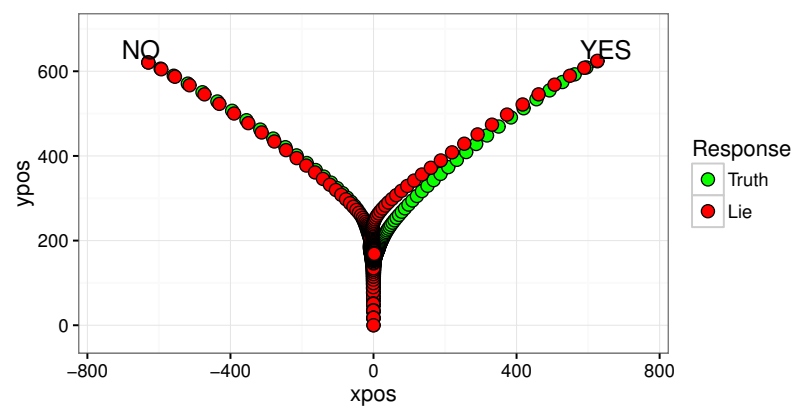

Fig. 6. Effects of Deception and Answer on Average Lie2Me Trajectories

Fool Me Once - Starting Position

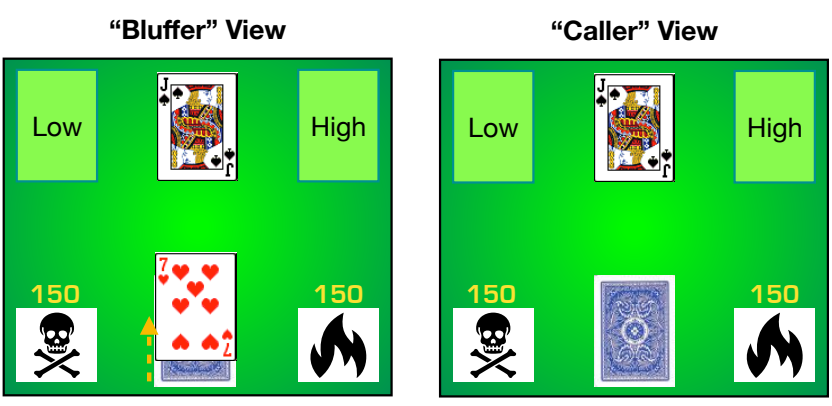

Fig. 7. Starting Position in a Fool Me Once! trial

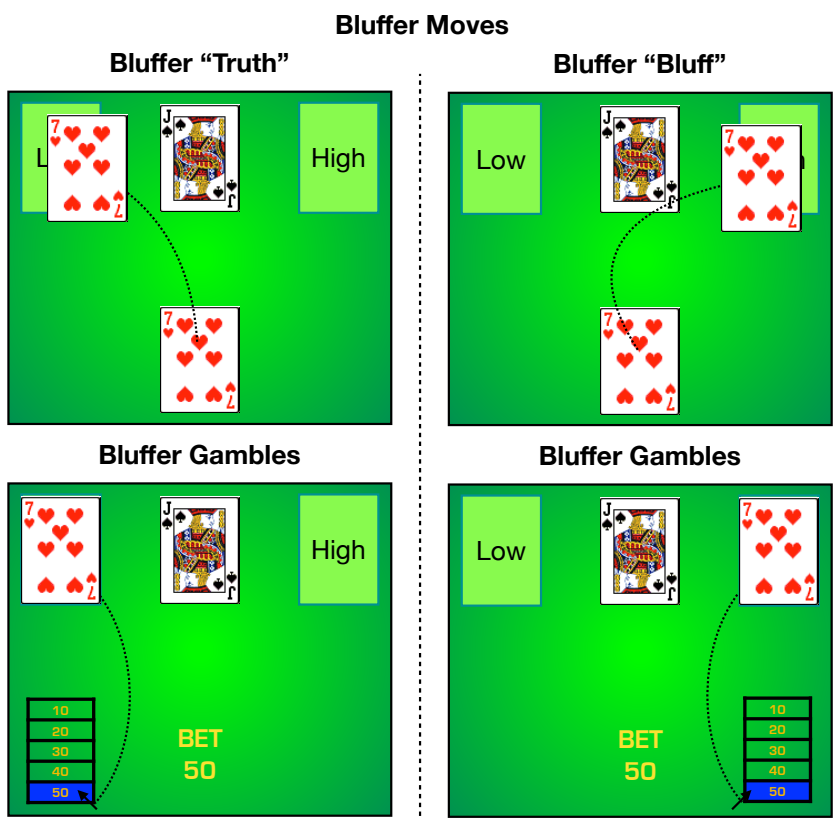

Fig. 8. Bluffer moves in a Fool Me Once! trial

"bluffer" (alternatively, "player") and "caller".

At the start of each turn, the bluffer and caller see one card at the top of screen, which is at the center and face up, and a second card, at the bottom of the screen, which is face down (Figure 7).

Next, the bluffer moves. The bluffer selects the card at the
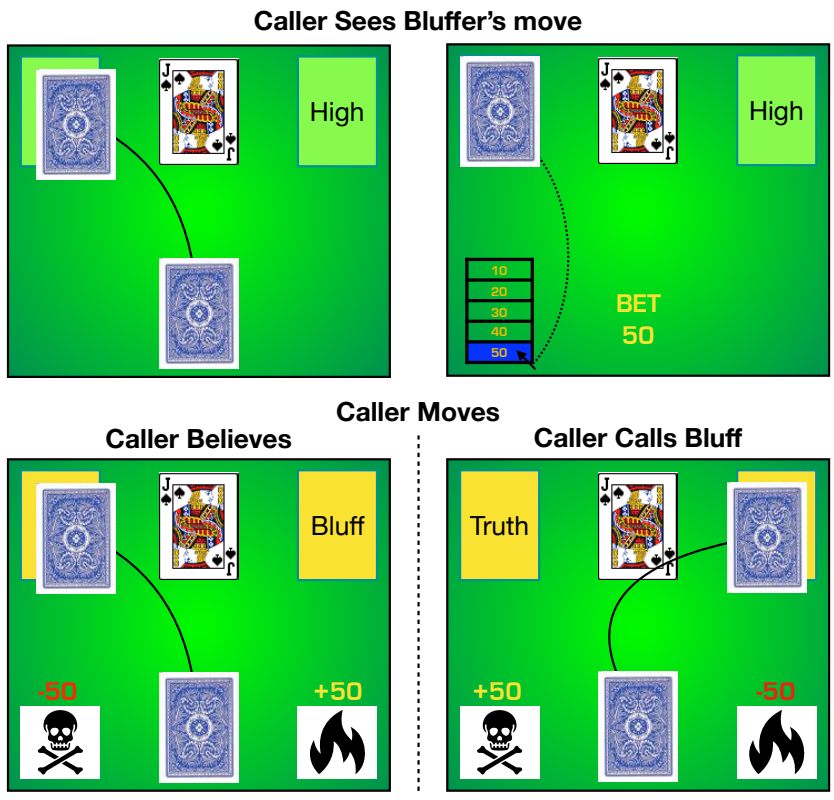

Fig. 9. Caller moves in a Fool Me Once! trial

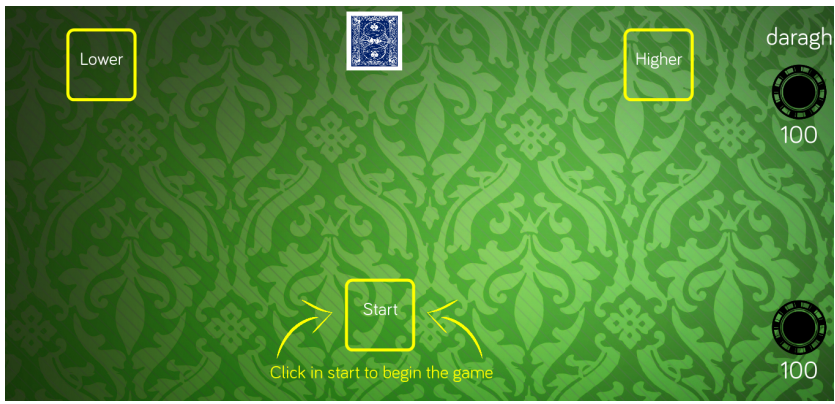

Fig. 10. Screenshot from Fool Me Once!

bottom of the screen and flips it face up in his or her view of the screen while the card remains face down for the caller. The bluffer then drags and drops the the visible face card to the right or left of the top center card to declare that his or her card is higher/lower than the center card. The caller on their screen observes in real time how the card is moved by the bluffer, but the card is still shown face down. The bluffer then bets a certain amount on their play (Figure 8).

The caller then calls whether they think the bluffer placed the bluffer card truthfully or bluffed (supposedly taking into account the observed bluffer's card movement trajectory). The caller places their face-down card to the right or left of the center card to declare the bluffer's move a truth or a bluff. (Figure 9)

3) Software: HTML5 with Javascript was used for the front-end of Fool Me Once!, with realtime client-serverclient communications using websockets and a custom-written node.js server. User profiles, leaderboards, and game statistics were stored on the server in a MongoDB database. Figure 10 provides a screenshot of the start of the game from the bluffer's perspective. 


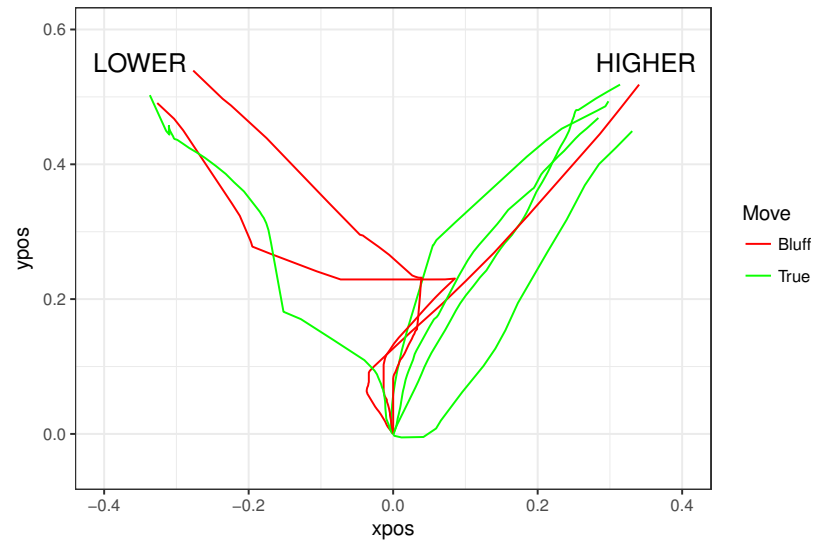

Fig. 11. Example trajectories of the "bluff" and "true" moves by the Bluffer from Fool Me Once!

\section{B. Results - Fool Me Once!}

Pilot participants were recruited to play Fool Me Once! in a computer lab to extract preliminary data. Examples of bluffer trajectories are provided in Figure 11. We need to be careful not to over-interpret these data, but there are indications that, similarly to Lie2Me, bluff trajectories are more deflected and more complex than truth trajectories. There also seems to be a bias towards picking "Higher" as an option, but it should be noted that we have not controlled for the value of the center card when selecting these trajectories.

\section{Discussion}

We have described two simple deception-focused games that track and record players' movements. Both paradigms highlight the potential of in-game movements as a source of information on the psychological processes of deception. Our first paradigm, Lie $2 \mathrm{Me}$, provided a wealth of data in a simple design similar to a laboratory experiment. We included approximately 300 participants in our analyses, but these data were collected in the first month of the exhibition and we anticipate obtaining 1000 participants or more. Obtaining such numbers of participants is far beyond what is possible in a laboratory experiment and allows a variety of analytic solutions (e.g., machine learning methods) that would not be possible to implement with lower numbers. Our early analyses of Lie2Me replicated well-established findings in the psychological literature in that movement during deception was slower, more deflected and more complex, but this finding was mostly restricted to affirmations (i.e., "yes" responses).

The second paradigm, Fool Me Once!, is an online twoplayer bluffing card game, and we are currently refining elements of the design and recruiting players. If we are successful in attracting and retaining players, the design of Fool Me Once! has considerable potential to further our understanding of deception. Most importantly, deception in Fool Me Once! arises spontaneously and naturally, so it potentially provides much greater insight into real-world deception. However, the design of Fool Me Once! also allows us to learn much about how and when humans detect deception. Based on our early analyses, it is likely that movement during bluffing is different from movement during truths. If so, it remains to be seen whether successful deception detectors are sensitive to such movement cues. With sufficient data, it would be possible to estimate the degree to which successful detectors exploit movement information of their opponents.

A focus on player movement may garner immediate benefits for game design. Crowdsourced player telemetry is an oftoverlooked source of data with which to train believable Artificial Intelligence (AI)-controlled agents, despite the fact that creating believable agents has been a longstanding goal of games AI [35], [36]. Limited previous work describes measurement of player behavior patterns and both quantitative and qualitative measures of player experience and engagement with various parts of the game (e.g., [36], [37], [38], [39]). The insights into the expression of deception in movement that we can glean from player movement will facilitate work towards computer-controlled adversaries who behave realistically when being deceptive, both in sensing deception from other players and generating their own deceptive moves.

An important avenue for future work will be to apply nonlinear machine learning models to analyze the patterns of deception in players' movements. Previous research suggests that in the simple games similar to the ones presented here, the data provided by recorded in-game movement can potentially enable accurate prediction of response truthfulness [30]. In the current preliminary analysis we focused on linear analysis of few simple measures characterizing overall response times, vacillation and movement deflection. Future investigations of players' movements in simple games similar to the ones presented here will explore the predictive value of more fine-grained measures based, e.g., on temporal and spatial properties of movement velocity and acceleration, as well as dynamical-systems-theoretic and information-theoretic modelbased measures [40], [41].

Overall, our work suggests that in-game movement provides an important source of data on active human psychological processes. We believe that the intersection between game design and cognitive science is an under-appreciated area of opportunity. Games enrich our lives with fun and entertainment and many have seen the potential in gamifying health and financial tasks. However, to date, there has been little consideration of the deep understanding of human psychology that can be extracted from in-game behavior, except to improve in-game experience. Crowdsourced player telemetry has the potential to provide rich longitudinal data on cognition and decision making in a variety of emotional contexts. Greater understanding of human behavior may help us improve mental health and games may even provide a vector for the administration of milder forms of psychotherapeutic intervention. Improving our understanding of human behavior will also enable the development of more credible artificial agents. Such agents will make games more entertaining, but they also have a wide variety of uses in the society of the future. 


\section{ACKNOWLEDGMENT}

The authors would like to thank Joanna Crispell, Derek Williams and all the team at Science Gallery Dublin ${ }^{\mathrm{TM}}$, Trinity College Dublin.

\section{REFERENCES}

[1] G. Calleja, "Revising Immersion: A Conceptual Model for the Analysis of Digital Game Involvement." in DiGRA Conference, 2007.

[2] M. S. El-Nasr, A. Drachen, and A. Canossa, Game analytics. Springer, 2016.

[3] M. A. Riley, K. Shockley, and G. V. Orden, "Learning From the Body About the Mind," Topics in Cognitive Science, vol. 4, pp. 21-34, 2012.

[4] R. F. Port and T. Van Gelder, Mind as motion: Explorations in the dynamics of cognition. The MIT Press, 1995.

[5] M. J. Spivey and R. Dale, "Continuous Dynamics in Real-Time Cognition," Current Directions in Psychological Science, vol. 15, no. 5, pp. 207-211, 2006.

[6] G. C. V. Orden, J. G. Holden, and M. T. Turvey, "Self-Organization of Cognitive Performance," Journal of Experimental Psychology: General, vol. 132, no. 3, pp. 331-350, 2003.

[7] C. Mckinstry, R. Dale, and M. J. Spivey, "Action Dynamics Reveal Parallel Competition in Decision Making," Psychological Science, pp. 17-20, 2008.

[8] D. Gilbert, "How Mental Systems Believe," American psychologist, vol. 46, no. 2, pp. 107-119, 1991.

[9] B. M. DePaulo, D. A. Kashy, S. E. Kirkendol, M. M. Wyer, and J. A. Epstein, "Lying in everyday life." Journal of Personality and Social Psychology, vol. 70, no. 5, pp. 979-995, 1996.

[10] K. B. Serota and T. R. Levine, "A Few Prolific Liars," Journal of Language and Social Psychology, vol. 34, no. 2, pp. 138-157, 2015.

[11] A. Eriksson and F. Lacerda, "Charlatanry in forensic speech science: A problem to be taken seriously," International Journal of Speech, Language and the Law, no. 2, pp. 169-193, 2007.

[12] N. Schilling and A. Marsters, "Unmasking Identity: Speaker Profiling for Forensic Linguistic Purposes," Annual Review of Applied Linguistics, vol. 35 , pp. 195-214, 2015 .

[13] E. H. Meijer, B. Verschuere, M. Gamer, H. Merckelbach, and G. BenShakhar, "Deception detection with behavioral, autonomic, and neural measures: Conceptual and methodological considerations that warrant modesty," Psychophysiology, vol. 53, no. 5, pp. 593-604, 2016.

[14] S. L. Sporer, "Deception and cognitive load: Expanding our horizon with a working memory model," Frontiers in Psychology, vol. 7, pp. 1-12, 2016.

[15] J. J. Walczyk, L. L. Harris, T. K. Duck, and D. Mulay, "A socialcognitive framework for understanding serious lies: Activation-decisionconstruction-action theory," New Ideas in Psychology, vol. 34, no. 1, pp. 22-36, 2014.

[16] C. S. Carter, T. S. Braver, D. M. Barch, M. M. Botvinick, D. Noll, and J. D. Cohen, "Anterior cingulate cortex, error detection, and the online monitoring of performance." Science, vol. 280, no. 5364, pp. 747-9, 1998.

[17] H. Garavan, T. J. Ross, K. Murphy, R. A. P. Roche, and E. A. Stein, "Dissociable Executive Functions in the Dynamic Control of Behavior : Inhibition, Error Detection, and Correction," NeuroImage, vol. 1829, pp. 1820-1829, 2002.

[18] T. S. Braver, J. R. Reynolds, and D. I. Donaldson, "Neural mechanisms of transient and sustained cognitive control during task switching," Neuron, vol. 39, no. 4, pp. 713-726, 2003.

[19] S. E. Christ, D. C. Van Essen, J. M. Watson, L. E. Brubaker, and K. B. McDermott, "The contributions of prefrontal cortex and executive control to deception: Evidence from activation likelihood estimate metaanalyses," Cerebral Cortex, vol. 19, no. 7, pp. 1557-1566, 2009.

[20] J. M. Nunez, B. J. Casey, T. Egner, T. Hare, and J. Hirsch, "Intentional false responding shares neural substrates with response conflict and cognitive control," Neuroimage, vol. 25, pp. 267-277, 2005.

[21] M. M. Botvinick, T. S. Braver, D. M. Barch, C. S. Carter, and J. D. Cohen, "Conflict Monitoring and Cognitive Control," Psychological Review, vol. 108, no. 3, pp. 624-652, 2001.

[22] J. M. C. Vendemia, R. F. Buzan, E. P. Green, S. The, A. Journal, N. Fall, and E. P. Green, "Practice effects, workload, and reaction time in deception," The American journal of psychology, vol. 118, no. 3, pp. 413-429, 2018.
[23] B. Verschuere, K. Suchotzki, and E. Debey, "Detecting Deception Through Reaction Times," in Detecting Deception. Chichester, UK: John Wiley \& Sons, Ltd, 2014, pp. 269-291.

[24] K. Suchotzki, B. Verschuere, B. V. Bockstaele, G. Ben-Shakhar, and G. Crombez, "Lying takes time: A meta-analysis on reaction time measures of deception," Psychological Bulletin, vol. 143, no. 4, pp. 428453, 2017.

[25] G. C. Van Orden and K. R. Paap, "Functional Neuroimages Fail to Discover Pieces of Mind in the Parts of the Brain," Philosophy of Science, vol. 64, pp. S85-S94, 1997.

[26] A. Gregg, "When Vying reveals Lying: The Timed Antagonistic Response Alethiometer," Applied Cognitive Psychology, vol. 21, pp. 621647, 2007.

[27] J. J. Walczyk, K. S. Roper, E. Seemann, and A. M. Humphrey, "Cognitive mechanisms underlying to questions: Response time as a cue to deception," Applied Cognitive Psychology, vol. 17, no. 7, pp. 755-774, 2003.

[28] N. D. Duran, R. Dale, and D. S. McNamara, "The action dynamics of overcoming the truth," Psychonomic Bulletin \& Review, vol. 17, no. 4, pp. 486-491, 2010.

[29] J. S. Valacich, J. L. Jenkins, S. Hariri, and J. Howie, "Identifying insider threats through monitoring mouse movements in concealed information tests," Hawaii International Conference on System Sciences in Jensen, M., Meservy, T., Burgoon, J., and Nunamaker, J., Report of the HICSS46 Rapid Screening Technologies, Deception Detection and Credibility Assessment Symposium, January 2013, p. 114, 2013.

[30] M. Monaro, L. Gamberini, and G. Sartori, "The detection of faked identity using unexpected questions and mouse dynamics," PLOS ONE, vol. 12 , no. 5 , pp. $1-19,2017$.

[31] D. O‘Hora, R. Carey, A. Kervick, D. Crowley, and M. Dabrowski, "Decisions in motion: decision dynamics during intertemporal choice reflect subjective evaluation of delayed rewards," Scientific reports, vol. 6, p. 20740, 2016.

[32] P. J. Kieslich and F. Henninger, "Mousetrap: An integrated, open-source mouse-tracking package," Behavior research methods, vol. 49, no. 5, pp. $1652-1667,2017$.

[33] H. Wickham, ggplot2: elegant graphics for data analysis. Springer, 2016.

[34] R. Dale, C. Kehoe, and M. J. Spivey, "Graded motor responses in the time course of categorizing atypical exemplars," Memory \& Cognition, vol. 35, no. 1, pp. 15-28, 2007.

[35] G. N. Yannakakis and J. Togelius, "A panorama of artificial and computational intelligence in games," IEEE Transactions on Computational Intelligence and AI in Games, vol. 7, no. 4, pp. 317-335, 2015.

[36] S. Redfern, "Player-Traced Empirical Cost-Surfaces for A* Pathfinding," in GAME'ON International Conference on Intelligent Games and Simulation. Eurosis, 2011

[37] J. Orkin and D. Roy, "The restaurant game: Learning social behavior and language from thousands of players online," Journal of Game Development, vol. 3, no. 1, pp. 39-60, 2007.

[38] S. Carter, M. Smith, S. Bali, S. Sotiriadis, N. Bessis, and R. Hill, "The use of Crowdsourcing to aid Quest Design in Games," in Emerging Intelligent Data and Web Technologies (EIDWT), 2012 Third International Conference on. IEEE, 2012, pp. 302-305.

[39] A. R. Gagné, M. Seif El-Nasr, and C. D. Shaw, "Analysis of telemetry data from a real-time strategy game: A case study," Computers in Entertainment (CIE), vol. 10, no. 1, p. 2, 2012.

[40] A. Zgonnikov, A. Aleni, P. Piiroinen, D. O'Hora, and M. di Bernardo, "Decision landscapes: visualizing mouse-tracking data," Royal Society open science, vol. 4, no. 11, p. 170482, 2017.

[41] A. Calcagnì, L. Lombardi, and S. Sulpizio, "Analyzing spatial data from mouse tracker methodology: An entropic approach," Behavior research methods, vol. 49, no. 6, pp. 2012-2030, 2017. 